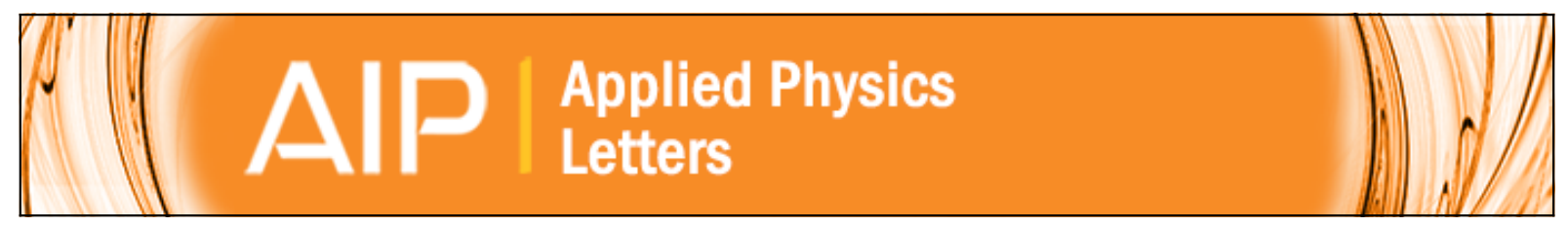

\title{
Luminescent properties and reduced dimensional behavior of hydrothermally prepared
} Y 2 Si 05 : Ce nanophosphors

D. W. Cooke, J.-K. Lee, B. L. Bennett, J. R. Groves, L. G. Jacobsohn, E. A. McKigney, R. E. Muenchausen, M. Nastasi, K. E. Sickafus, M. Tang, J. A. Valdez, J.-Y. Kim, and K. S. Hong

Citation: Applied Physics Letters 88, 103108 (2006); doi: 10.1063/1.2183737

View online: http://dx.doi.org/10.1063/1.2183737

View Table of Contents: http://scitation.aip.org/content/aip/journal/apl/88/10?ver=pdfcov

Published by the AIP Publishing

\section{Articles you may be interested in}

Photoluminescence phenomena of Ce 3 +-doped Y 3 Al 5 O 12 nanophosphors

J. Appl. Phys. 102, 083541 (2007); 10.1063/1.2798513

Vacuum ultraviolet-ultraviolet and x-ray excited luminescence properties of Ba $3 \mathrm{Gd}$ ( B O 3 ) 3 : Ce 3 + J. Appl. Phys. 101, 113530 (2007); 10.1063/1.2743826

Enhanced luminescence of Y 3 Al 5 O 12 : Ce 3 + nanophosphor for white light-emitting diodes Appl. Phys. Lett. 89, 173118 (2006); 10.1063/1.2367657

Low-temperature upconversion spectroscopy of nanosized Y $2 \mathrm{O} 3: \mathrm{Er}, \mathrm{Yb}$ phosphor J. Appl. Phys. 98, 063529 (2005); 10.1063/1.2058195

Green phosphorescence of $\mathrm{CaAl} 2 \mathrm{O} 4: \mathrm{Tb} 3+$, Ce 3+ through persistence energy transfer Appl. Phys. Lett. 80, 1535 (2002); 10.1063/1.1456955

\section{NEW! Asylum Research MFP-3D Infinity ${ }^{\text {Tm }}$ AFM} Unmatched Performance, Versatility and Support

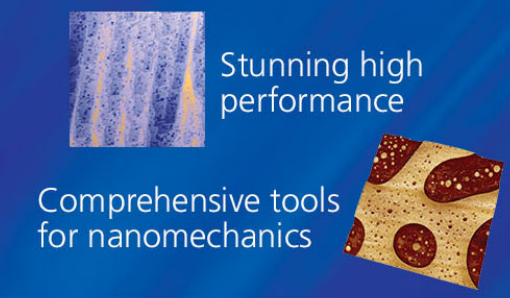

Simpler than ever to GetStarted ${ }^{\mathrm{T}}$

Widest range of accessories

for materials science and bioscience

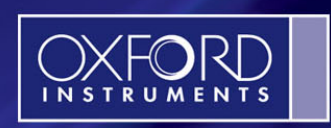

The Business of Science ${ }^{2}$ 


\title{
Luminescent properties and reduced dimensional behavior of hydrothermally prepared $\mathrm{Y}_{2} \mathrm{SiO}_{5}$ : Ce nanophosphors
}

\author{
D. W. Cooke, ${ }^{\text {a) }}$ J.-K. Lee, B. L. Bennett, J. R. Groves, L. G. Jacobsohn, E. A. McKigney, \\ R. E. Muenchausen, M. Nastasi, K. E. Sickafus, M. Tang, and J. A. Valdez \\ Materials Science and Technology Division, Los Alamos National Laboratory, Los Alamos, \\ New Mexico 87545 \\ J.-Y. Kim and K. S. Hong \\ School of Materials Science and Engineering, Seoul National University, Shillim-dong, Seoul 151-744, \\ South Korea
}

(Received 13 September 2005; accepted 7 February 2006; published online 7 March 2006)

\begin{abstract}
Hydrothermally prepared nanophosphor $\mathrm{Y}_{2} \mathrm{SiO}_{5}$ : Ce crystallizes in the $\mathrm{P} 2{ }_{1} / \mathrm{c}$ structure, rather than the B2/b structure observed in bulk material. Relative to bulk powder, nanophosphors of particle size $\sim 25-100 \mathrm{~nm}$ diameter exhibit redshifts of the photoluminescence excitation and emission spectra, reduced self absorption, enhanced light output, and medium-dependent radiative lifetime. Photoluminescence data are consistent with reduced symmetry of the $\mathrm{P} 2{ }_{1} / \mathrm{c}$ structure and are not necessarily related to reduced dimensionality of the nanophosphor. In contrast, medium-dependent lifetime and enhanced light output are attributed to nanoscale behavior. Perturbation of the Ce ion electric field is responsible for the variable lifetime. (C) 2006 American Institute of Physics.
\end{abstract}

[DOI: $10.1063 / 1.2183737$ ]

Motivated by the intriguing optical properties that result from reduced dimensionality in inorganic insulators, ${ }^{1}$ and the important application of rare earth $(R E)$-doped oxyorthosilicates $\mathrm{Lu}_{2} \mathrm{SiO}_{5}: \mathrm{Ce}$ and $\mathrm{Y}_{2} \mathrm{SiO}_{5}: \mathrm{Ce}$ (YSO) to radiation detection, ${ }^{2}$ we have synthesized and investigated the optical behavior of nanoscale YSO. From the data, we identify those properties that can be directly attributed to reduced dimensionality.

Nanophosphor YSO ( $n$-YSO) was prepared by the hydrothermal method using reagent-grade $\mathrm{Y}\left(\mathrm{NO}_{3}\right)_{3} \cdot 6 \mathrm{H}_{2} \mathrm{O}$, $\mathrm{Ce}\left(\mathrm{NO}_{3}\right)_{3} \cdot 6 \mathrm{H}_{2} \mathrm{O}$ and $\mathrm{SiO}_{2}$ of $>99 \%$ purity. Powders were mixed with deionized water and titrated to $\mathrm{pH}$ of 9 and then aged in a hydrothermal reactor at $180{ }^{\circ} \mathrm{C}$ for $24 \mathrm{~h}$ to nucleate nanocrystals. The mixture was dried and the resulting powder was annealed at $1000{ }^{\circ} \mathrm{C}$ in oxygen to promote chemical ordering and growth of $n$-YSO. The cerium content of the resulting specimen was 1 at. $\%$.

Figure 1 shows the x-ray diffraction (XRD) pattern of $n$-YSO, which consists primarily ( $\sim 95 \%$ by volume) of the monoclinic phase with space group $\mathrm{P} 2{ }_{1} / \mathrm{c}\left(X_{1}\right.$ structure $)$. These results are consistent with previous observations where it was shown that for synthesis temperatures less than $\sim 1100{ }^{\circ} \mathrm{C}$, YSO crystallizes in the monoclinic structure with space group $\mathrm{P} 2{ }_{1} / \mathrm{c}^{3}$ This latter structure has two inequivalent $R E$ sites coordinated with seven and nine oxygens whereas the $\mathrm{B} 2 / \mathrm{b}$ structure $\left(X_{2}\right.$ observed in bulk material) is six and seven oxygen coordinated. Reference data for the $X_{1}$ and $X_{2}$ structures are shown as solid and dotted stick spectra, respectively.

Nanophosphor YSO microstructure was revealed through transmission electron microscopy (TEM). Figure 2(a) is a TEM micrograph showing typical large and small particles; Grains A and B have dimensions $\sim 100$ and $25 \mathrm{~nm}$, respectively. This image was obtained in dark-field condi-

\footnotetext{
a) Author to whom correspondence should be addressed; electronic mail:
} cooke@lanl.gov tions wherein a portion of the first- and second-order rings in the diffraction pattern was used to produce diffraction contrast in the image, thus highlighting the nanocrystals. Figure 2(b) shows a selected area diffraction pattern obtained from the sample region shown in Fig. 2(a) indicating that the material possesses a high degree of crystallinity in agreement with the XRD results.

Photoluminescence emission (PL) and excitation (PLE) spectra of bulk and $n$-YSO were obtained with a Photon Technology International TimeMaster ${ }^{\mathrm{TM}}$ steady-state fluorimeter and lifetime spectrometer. The detector slits are orthogonal to the excitation beam (120 W Xe lamp) and are located $20 \mathrm{~cm}$ from the sample. The nanophosphor PL spectrum (dotted line) exhibits a well-defined maximum at $431 \mathrm{~nm}\left(\lambda_{\mathrm{ex}}=366 \mathrm{~nm}\right)$ along with a knee near $470 \mathrm{~nm}$, and is redshifted relative to the bulk spectrum (solid line) as shown in Fig. 3. The spectrum exhibits no time dependent shifts. The broad spectrum of the bulk sample consists of peaks at 395 and $420 \mathrm{~nm}\left(\lambda_{\mathrm{ex}}=356 \mathrm{~nm}\right)$, which are attributed

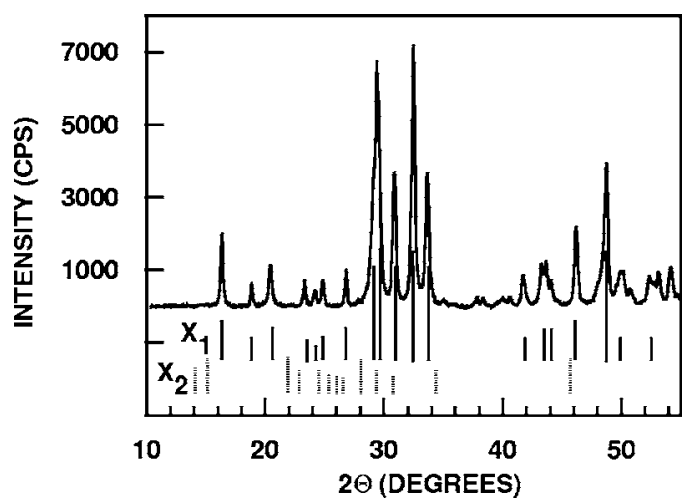

FIG. 1. X-ray diffraction pattern of $n$-YSO showing that it crystallizes in the $X_{1}$ structure. Principal diffraction peaks of the $X_{1}$ and $X_{2}$ structures are shown as solid and dotted stick spectra, respectively (Reference data correspond to JCPDF cards 41-004 and 74-1266). 

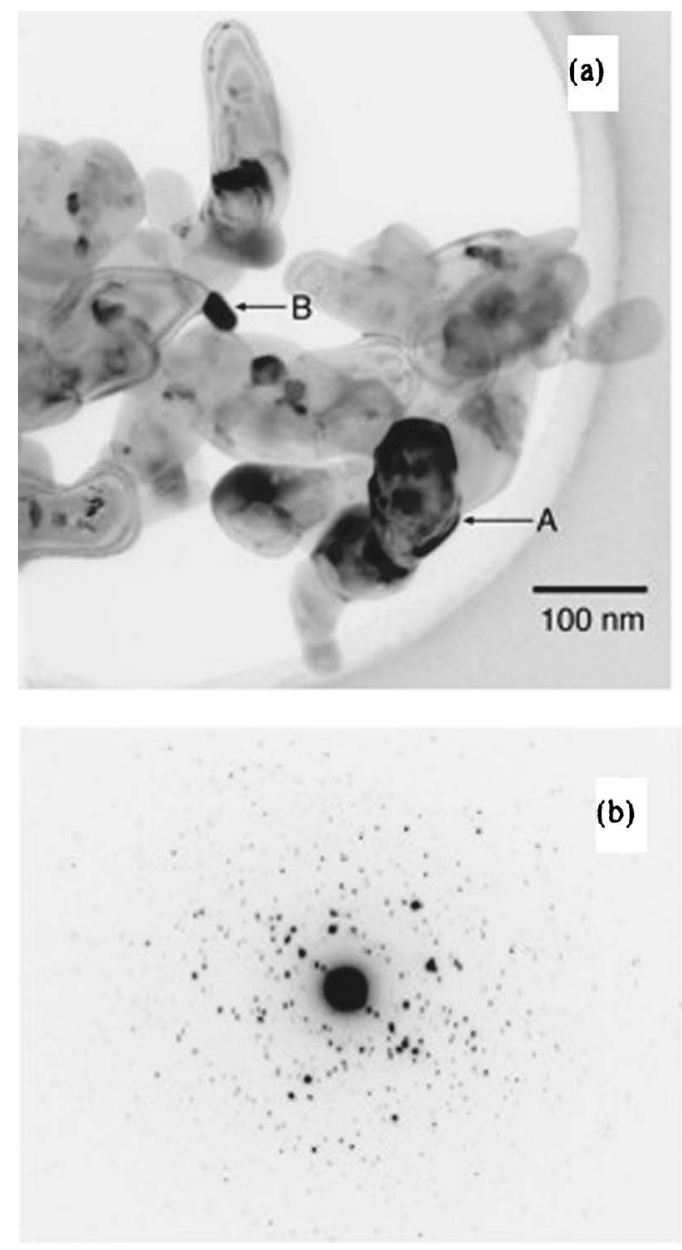

FIG. 2. (a) TEM micrograph of $n$-YSO showing typical (A) large (100 nm) and (B) small $(25 \mathrm{~nm})$ grains. (b) Selected area diffraction pattern obtained from the sample region shown in (a), which indicates a high degree of crystallinity.

to the well-known emission from the spin-orbit split ground state of the $\mathrm{Ce}^{3+} 4 f$ electron manifold. ${ }^{4}$ Overlap of the excitation and emission bands is greater in the bulk material than in the nanophosphor implying increased self absorption of the bulk specimen.

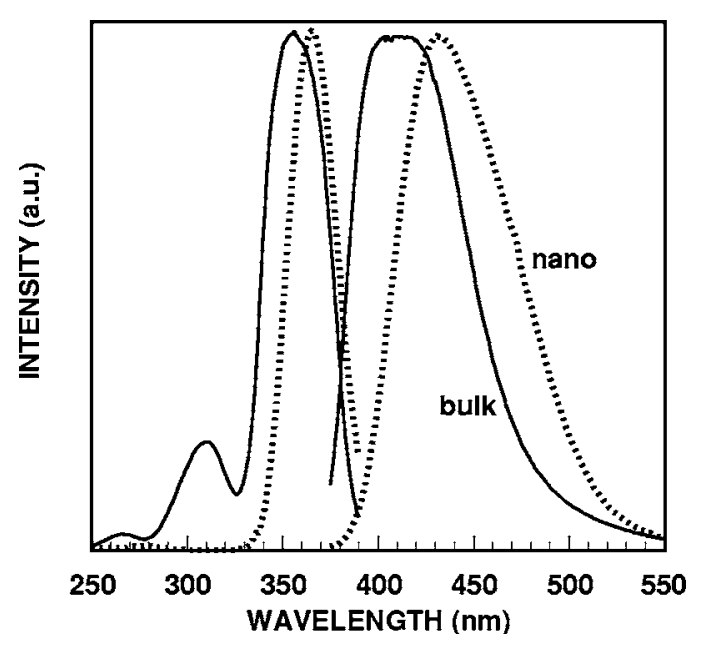

FIG. 3. PL and PLE spectra of bulk (solid lines) and $n$-YSO (dotted lines). Data have been normalized for illustrative purposes. Note the enhanced overlap of the bulk excitation and emission bands relative to the nanophosphor bands.
The bulk PLE spectrum of YSO is characterized by $\mathrm{Ce}^{3+}$ excitation bands at 356, 310, and $265 \mathrm{~nm}$, which are associated with the crystal-field split $\mathrm{Ce}^{3+} 5 d$ electronic levels. The $n$-YSO spectrum exhibits only the main excitation peak $(366 \mathrm{~nm})$ of the $\mathrm{Ce}^{3+}$ ion and is redshifted relative to the bulk peak. At issue is the origin of the PL and PLE peak shifts: Are they due to reduced dimensionality and concomitant large surface-to-volume ratio of the nanophosphors or are they simply due to different crystal symmetries? Macroscopic $\mathrm{Gd}_{2} \mathrm{SiO}_{5}$ : Ce crystallizes in the monoclinic structure with $\mathrm{P} 2{ }_{1} / \mathrm{c}$ symmetry and exhibits nearly identical PL and PLE spectra as we observe in $n$-YSO. ${ }^{5}$ It would be extremely fortuitous if reduced dimensionality in a very similar oxyorthosilicate with the same crystal structure and point symmetry yielded these results. We conclude that it is more plausible that PL and PLE of $n$-YSO with particle sizes $25-100 \mathrm{~nm}$ are primarily determined by the activator ion symmetry rather than reduced dimensionality.

However, reduced dimensionality effects are manifested in the lifetime measurements of $n$-YSO. Ambient optical excitation of the main PLE bands of bulk and $n$-YSO powder yields single exponential fits with $1 / e$ values of 39 and $55 \mathrm{~ns}$, respectively. Excitation is provided by a dye-pumped $\mathrm{N}_{2}$ laser of $0.8 \mathrm{~ns}$ pulse width, $0.2 \mathrm{~mJ} /$ pulse energy, and $5 \mathrm{~Hz}$ rate. A convenient method for measuring lifetime of powder specimens is to suspend the particles in a solvent and magnetically stir them during optical excitation. In conducting this experiment we found, quite surprisingly, that the nanophosphor lifetime was dependent upon the medium in which it was immersed whereas the bulk YSO was independent of the medium. Nanophosphor YSO lifetimes were measured in air (index of refraction $n=1.0)$, ethanol $(n=1.32)$, methanol $(n=1.32)$, deionized water $(n=1.33)$, dimethylformamide $(n=1.43)$, and epoxy $(n=1.54)$, and yielded fitted lifetimes 55.0, 44.0, 43.2, 43.5, 40.0, and $35.0 \mathrm{ns,} \mathrm{respec-}$ tively. Typical error bar on these measurements is $\pm 3 \%$.

Cerium emission in YSO is dominated by parity-allowed electric dipole transitions and for a one-electron system the inverse radiative lifetime for transitions between initial and final states is given by ${ }^{6}$

$$
\frac{1}{\tau_{i j}}=\frac{n\left(n^{2}+2\right)^{2}}{9} \frac{2 \pi e^{2} f_{i j}}{\varepsilon_{0} m c \lambda^{2}}
$$

with emission wavelength $\lambda$ and oscillator strength $f_{i j}$. The first fraction in Eq. (1) is a correction to the local electric field seen by the Ce ion, which differs from the macroscopic electric field. In micron-size YSO, most Ce ions are far from the surface and their dielectric interaction with the embedding medium is negligible; therefore, $n$ is simply the refractive index of the host lattice, viz. 1.82. In contrast, nanoscale YSO has a much larger surface-to-volume ratio with most of the ions residing near the surface. Consequently, the dopant Ce ions now experience a local electric field induced by the surrounding medium, which is different from the average field found in bulk YSO. Similar observations have been reported for $\mathrm{Y}_{2} \mathrm{O}_{3}:$ Eu. ${ }^{7}$ Therefore, the index of refraction to be used in Eq. (1) is not the value of YSO but the value of the surrounding medium. Figure 4 is a plot of $\tau^{-1} v s$. $n\left(n^{2}+2\right)^{2}$ for the various media. Assuming constant oscillator strength, there is excellent agreement between Eq. (1) and experiment. This approach provides a straightforward method for measuring osciliator strengths of optical transi- 


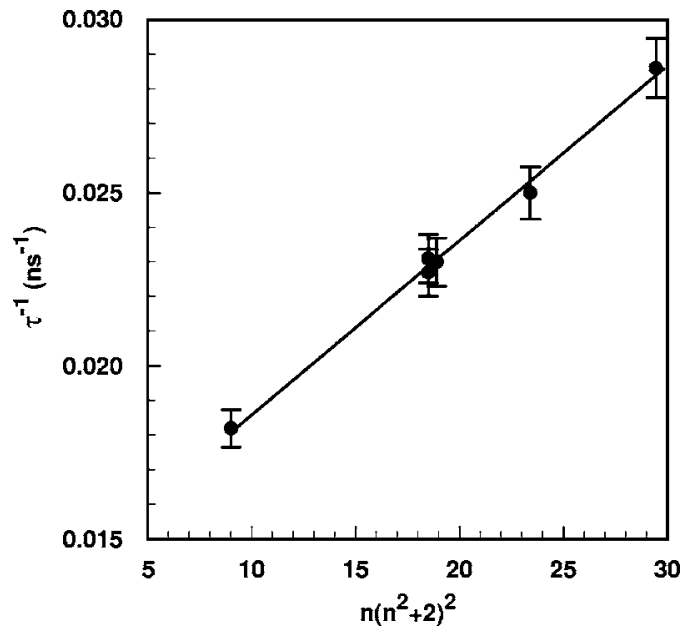

FIG. 4. Inverse radiative lifetime as a function of index of refraction for $n$-YSO embedded in various media with refractive index ranging from 1.0 to 1.54 .

tions in nanophosphors. Accordingly, we extracted $f_{i j}$ from the experimentally determined slope; the value is 0.013 , which is $\sim 50 \%$ greater than the bulk value ${ }^{8}$ and is consistent with enhanced light output as observed in this work. Finally, we note that although a solvent of index 1.82 was unavailable, extrapolation of the data in Fig. 4 shows $\tau=26$ ns for $n=1.82$, which is considerably shorter than the measured value in bulk (39 ns) and emphasizes the effect of reduced dimensionality on optical properties of nanomaterials.

Because $n$-YSO PL and PLE spectra are independent of the surrounding medium with only the lifetime affected, we conclude that perturbation of the local electric field due to reduced dimensionality is responsible for medium-dependent lifetime in $n$-YSO.

Additional reduced-dimension behavior is associated with enhanced light output of $n$-YSO excited by $x$ radiation. Under ultraviolet excitation the bulk and $n$-YSO intensities are nearly equivalent; however, when excited by $\mathrm{x}$ rays, the nanophosphor output is $\sim$ three times bulk when the data are normalized to mass. This large increase in light output cannot be solely explained by the different bulk and nanophosphor structures and their intrinsically different luminescent efficiencies. Data of Fig. 3 clearly indicate less selfabsorption of $n$-YSO relative to bulk, which will increase overall light output of the nanophosphor, but certainly not by a factor of 3 . Of course, photon scattering by particles of varying size will have a significant effect on luminescence intensity when measured by a fixed solid-angle detector as done in this experiment. For micron-size particles Mie scattering is expected to be the dominant mechanism whereas Rayleigh scattering should dominate for nm-size objects. The latter mechanism obeys a $\lambda^{-4}$ dependence on wavelength and thus is expected to reduce the measured light output of $n$-YSO. This cannot account for the large increase in $n$-YSO output relative to bulk YSO. Therefore, we tentatively conclude that enhanced light output from $n$-YSO originates from reduced dimensionality although the fundamental mechanism cannot be deduced from the present data. Nevertheless, enhanced light output with tunable lifetime offers intriguing possibilities for technical applications, especially radiation detectors.

This research was supported by Laboratory Directed Research and Development funds (\#20040213ER) and the DOE Office of Basic Energy Sciences (\#07SCPE972).

${ }^{1}$ T. Aitasalo, J. Holsa, M. Lastusaari, J. Nittykoski, and F. Pelle, Opt. Mater. (Amsterdam, Neth.) 27, 1511 (2005).

${ }^{2}$ M. J. Weber, Nucl. Instrum. Methods Phys. Res. A 527, 9 (2004).

${ }^{3}$ C. Cannas, M. Mainas, A. Musinu, G. Piccaluga, A. Speghini, and M. Bettinelli, Opt. Mater. (Amsterdam, Neth.) 27, 1506 (2005).

${ }^{4}$ G. Blasse and B. C. Grabmaier, Luminescent Materials (Springer, Berlin, 1994).

${ }^{5}$ H. Suzuki, T. A. Tombrello, C. L. Melcher, and J. S. Schweitzer, Nucl. Instrum. Methods Phys. Res. A 320, 263 (1992).

${ }^{6}$ B. Henderson and G. F. Imbusch, Optical Spectroscopy of Inorganic Solids (Clarendon, Oxford, 1989), p. 173.

${ }^{7}$ R. S. Meltzer, S. P. Feofilov, B. Tissue, and H. B. Yuan, Phys. Rev. B 60, R14012 (1999).

${ }^{8}$ D. W. Cooke, B. L. Bennett, K. J. McClellan, J. M. Roper, M. T. Whittaker, and A. M. Portis, Phys. Rev. B 61, 11973 (2000). 